\title{
HEPATIC ADENOMA MIMICKING A LEYDIG-SERTOLI CELL TUMOR METASTASIS
}

\author{
Thomas ROSSOR ${ }^{1}$, Alberto QUAGLIA ${ }^{2}$, Stergios ZACHAROULIS ${ }^{3}$, \\ Sandra STRAUTNIEKS ${ }^{2}$, Mark DAVENPORT ${ }^{1}$, Nedim HADZIC ${ }^{1,2}$
}

${ }^{1}$ Paediatric Liver Service, King's College Hospital, London, UK, ${ }^{2}$ Institute of Liver Studies, King's College Hospital, London, UK, ${ }^{3}$ Department of Paediatric Oncology Royal Marsden Hospital, Sutton, UK

Corresponding author:

Thomas Rossor

Paediatric Liver Service

King's College Hospital

Denmark Hill

London SE5 9RS, UK

trossor@nhs.net

Tel.: + 02032998495

Fax.: + 02032994228

Received: March 11, 2013

Accepted: April 22, 2013

Copyright (C) 2013 by University Clinical Center Tuzla. E-mail for permission to publish: paediatricstoday@ukctuzla.ba
Objective - A description of the co-occurence of two rare paediatric neoplasias, and a discussion of the possible pathogenic processes and potential role of a tumour disposition syndrome. Case report - We describe a 14 year old girl presenting with an abdominal mass which was found on resection to be a Leydig-Sertoli cell tumour. This was associated with a hepatic lesion suspected of a metastasis. Due to persistence despite chemotherapy the liver lesion was resected and confirmed to be a hepatocellular adenoma, providing the first reported cooccurrence of these rare neoplasms. Conclusion - Hormonal activity of a Sertoli-Leydig cell tumour may predispose to hepatic adenoma, or the coincidence of these two tumours may be accounted for by a tumour predisposition syndrome. When investigating liver lesions the potential for an adenoma to mimic a metastasis should be considered.

Key words: Adenoma - Liver cell - Sertoli Leydig cell tumor Hyperthyroidism.

\section{Introduction}

Leydig-Sertoli cell (LSC) tumours are a subset of the sex cord-stromal tumors, seen rarely in the paediatric population, that comprise less than $1 \%$ of ovarian malignancies. Three-quarters of LSC tumours present in the first three decades of life with the mean age at presentation between 25 and 30 years; more than half are hormonally active. Of those, around $30-40 \%$ produce androgens, while the remainder secrete other hormones including oestrogens. At diagnosis 95\% of these tumors are confined to the ovary (1).
Hepatocellular adenomas (HCA) are very rare in children, and there are no reliable incidence data. They are histologically heterogeneous, allowing their subclassification into four distinct categories: 1) HNF1a-mutated (30-50\% of HCA) which show marked steatosis and rarely progress to hepatocellular carcinoma (HCC), 2) B-Catenin positive (10$15 \%)$, commonly seen in men, with greater progression to HCC, and 3) HCA without these specific mutations either with $(35 \%)$ or without inflammatory changes (5-10\%)(2).

HCAs have been reported in the context of several chronic conditions including gly- 
cogen storage diseases and Fanconi anaemia. In the latter, the association of HCA with the use of therapeutic androgens has been noted (2). It is in the presence of exogenous hormones that HCAs have become increasingly prevalent in the young female population using the oral contraceptive pills (OCP) since the 1960s. HCA growth due to increased hormonal stimulation in pregnancy is also well recognised (3).

While there is extensive literature on the effects of exogenous hormones in the development of HCA, the role played by endogenous hormones is not as established. HCA has been reported in association with polycystic ovary syndrome (PCOS), with demonstrable abnormal sex hormone profiles (4).

We present the case of a HCA occurring in a teenage prepubertal girl with a history of ovarian LSC tumour. We discuss the possible relationships between the two neoplasms and the possibility that the abnormal endogenous hormonal secretion by the ovarian tumour could have provided a stimulus to the growth of the HCA.

\section{Case report}

A 14 year old girl presented with a short history of right-sided abdominal pain and swelling. Her past medical history was uneventful, except for recurrent urinary tract infections, prompting renal ultrasound (US) at 8 years, which was unremarkable. She had had no menarche.

On examination she had a significant ascites. Tanner 4 breast and pubic hair development was noted. Abdominal US and MRI imaging showed a heterogeneous multiloculated abdominal mass $(11 \times 10 \mathrm{~cm})$ with one well defined solid component $(5.3 \mathrm{~cm})$ arising from the left ovary and extending up to the level of the umbilicus. Ascites and a single nodule $(1.5 \mathrm{~cm})$ in segment VIII of the liver were also noted (see below). Laboratory results showed (normal values): full blood count, renal/bone/liver profile, and coagulation profile normal. A-fetoprotein $7 \mathrm{kIU} / \mathrm{l}$ (<7), $\beta$-human chorionic gonadotrophin 2 IU/l (<5), cancer antigen 125 (CA125) 219 $\mathrm{kU} / \mathrm{l}(<35)$. A hormonal profiling was not carried out at this stage. A laparotomy was performed and the ovarian mass resected. The liver was macroscopically normal. Upon histopathological examination the diagnosis of an intermediate grade SLC tumour was made. Removal of the liver mass was not attempted as it was felt that it would require expertise of a hepatobiliary surgeon. A positron emission tomography (PET) scan was performed postoperatively to evaluate the liver lesion and showed the presence of a solitary fludeoxyglucose (FDG) avid lesion within segment VIII with an SUV of 3.99, consistent with a metastatic deposit (Fig. 1).

Between the surgery and planned chemotherapy the girl underwent menarche and was started on a GnRH receptor agonist leuprorelin as prophylaxis to prevent bleeding secondary to chemotherapy-induced thrombocytopenia. Chemotherapy was commenced with cisplatin and etoposide as part of BEP regimen without bleomycin. Three cycles of chemotherapy were given at intervals of four weeks, but due to the radiological persistence of the hepatic lesion after three months, a

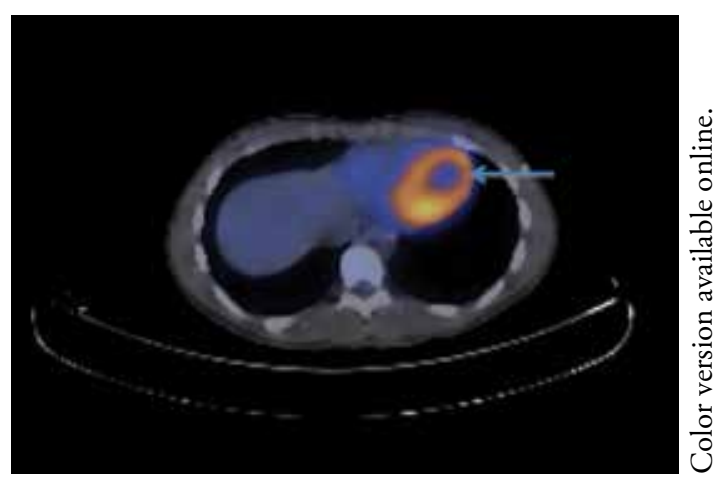

Fig. 1 PET scan following resection of ovarian tumour, showing solitary fludeoxyglucose avid lesion in segment VIII of the live. 


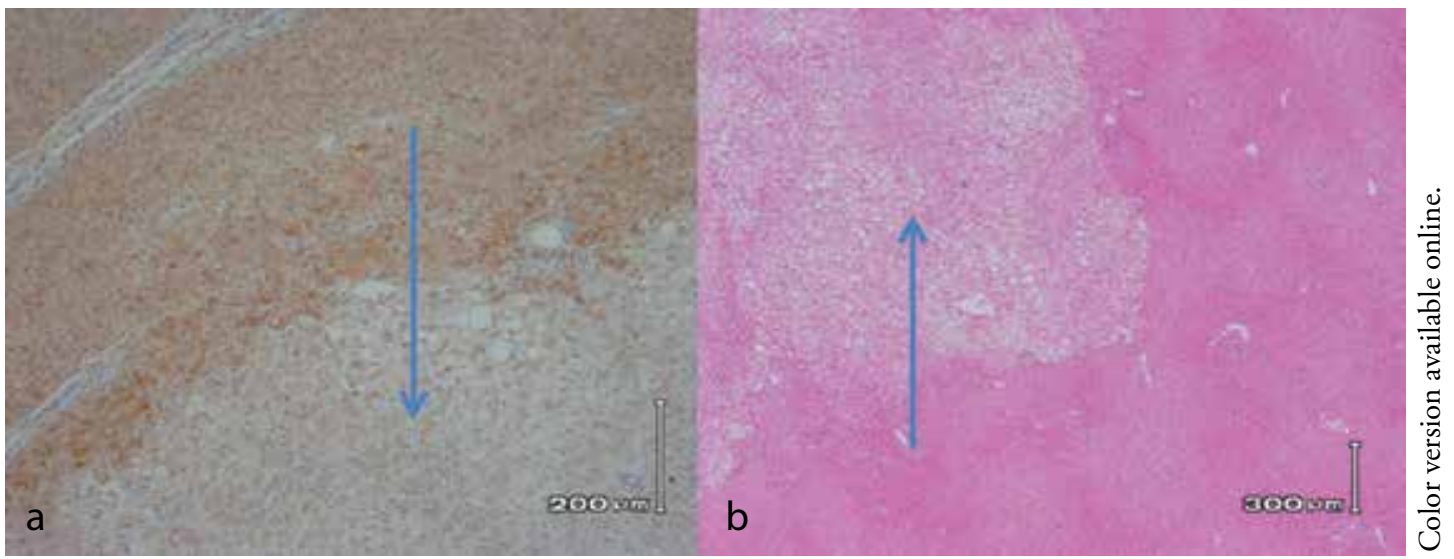

Fig. 2 A) Low magnification (40x, H\&E stain) view of lesional tissue and adjacent liver. The lesional tissue is well demarcated, acapsular and shows marked steatosis. B) On immunohistochemistry, the lesional hepatocytes do not stain for fatty acid binding protein (FABP), the expression of which is preserved in the non-steatotic adjacent parenchyma (100x).

further three cycles were administered uneventfully. Repeated PET scan showed no evidence of regression and it was decided to resect the liver lesion. At laparotomy external appearance of the liver was normal with no discernable tumour. Intraoperative US was used to localize the mass and a wire inserted into it aiding the subsequent segment VIII resection. The $10 \mathrm{~mm}$ lesion appeared as a yellowish nodule devoid of a capsule (Fig. 2).

Histopathologically, the overall appearance was of a benign hepatocellular lesion, favouring a HCA over focal nodular hyperplasia. Immunohistochemical profile of HCA was suggestive of the subcategory associated with mutation of the TCF1 locus, which encodes the hepatocyte nuclear factor 1a (HNF1a) transcription factor. However, in situ mutational analysis of the TCF1 locus was negative.

After the second surgery the girl complained of increasing lethargy and fatigue and was found to have an enlarged thyroid gland. Initial thyroid function tests were unremarkable. Four weeks later she reported tremors, poor sleep and agitation. Her repeated thyroid function tests revealed: TSH $<0.01$ $\mathrm{mU} / \mathrm{l}(0.38-4.7 \mathrm{mU} / \mathrm{l})$, free T4 $51 \mathrm{mmol} / \mathrm{l}$
(10-23 mmol/l), free T3 $15.7 \mathrm{mmol} / \mathrm{l}$ (3.7$7.9 \mathrm{mmol} / \mathrm{l})$ suggesting hyperthyroidism. US scan of the thyroid showed no nodules, and she was started on carbimazole. One year after the surgery she remains clinically well with normal liver and thyroid function and regular periods. Follow-up liver US showed no recurrent. lesions.

\section{Discussion}

We have described here previously unreported co-occurrence of ovarian LSC tumour with HCA in a 14 year old girl. LSC tumours typically present with features that include virilisation, dysmenorrhoea, abdominal pain and distension. Reported endocrine abnormalities include a raised serum testosterone in the virilized group, but varying levels of urinary 17 -ketosteroid. However, as with our patient, the majority of patients within the large series of SLC tumours did not have steroid profiles performed (1).

In adults the incidence of HCA rose significantly during the 1960s with the increasing use of the OCP (3). HCAs have similarly been frequently described in the context of exogenous androgen exposure. Hepatocytes 
have been shown to express both oestrogen and androgen receptors, although their role in normal liver is unclear (3). Excessive activation of these receptors may result in the observed lipogenesis, glycogenesis, while increased activity of other pathways [such as mammalian target of rapamycin (mTOR)] may play a role in the neoplastic change (5).

Abnormal hormonal stimulation could explain the co-occurrence of HCA with the LSC tumour which, though unfortunately not documented, was likely to be hormonally active at diagnosis. Indirect clinical evidence support for a role of an hormonal imbalance could be the primary amenorrhoea, followed by menarche following LSC tumour resection. In the absence of initial urine steroid profile this link remains speculative.

The incidence of metastases in intermediate grade SLC tumour is low, reported as $8 \%$ (1). It is noteworthy that the HCA of our patient has produced a positive signal on FDG PET imaging, whereas these lesions are typically expected to have low or normal FDG uptake. To our knowledge this unusual observation has been reported only once, (6) but should be borne in mind when using this modern technique to assess nature of focal hepatic lesions.

Thyroid abnormalities are recognized in association with ovarian tumours. A recent study examined a whole range of tumours to look at the role of DICER1 mutations as a pleotropic tumour predisposition syndrome (DICER1 syndrome). In two thirds of the SLC tumours the DICER1 mutation was found, and a high incidence of thyroid cyst/ goitre was observed in both probands and relatives (7). HCA has not yet been reported in this context.

\section{Conclusion}

In conclusion, HCA should be considered in the differential diagnosis of hepatic lesions synchronous or metachronous to extrahepatic primary neoplasms with hormone secretion potential, raising the question of potential pathogenetic links between these rare conditions.

Acknowledgement: Supported by the Paediatric Liver Service, King's College Hospital.

Authors' contributions: Conception and design: NH, TR; Acquisition, analysis and interpretation of data: AQ, SS; Drafting the manuscript: MD, NH, AQ, TR; Revising it critically for important intellectual content: MD, NH, AQ, TR, SS, SZ.

Conflict of interest: The authors declare that they have no conflict of interest.

\section{References}

1. Young RH, Scully RE. Ovarian Sertoli-Leydig cell tumors. A clinicopathological analysis of 207 cases. Am J Surg Pathol. 1985;9(8):543-69.

2. Rebouissou S, Bioulac-Sage P, Zucman-Rossi J. Molecular pathogenesis of focal nodular hyperplasia and hepatocellular adenoma. J Hepatol. 2008;48(1):163-70.

3. Cohen C, Lawson D, DeRose PB. Sex and androgenic steroid receptor expression in hepatic adenomas. Hum Pathol. 1998;29(12):1428-32.

4. Toso C, Rubbia-Brandt L, Negro F, Morel P, Mentha G. Hepatocellular adenoma and polycystic ovary syndrome. Liver Int. 2003;23(1):35-7.

5. Pelletier L, Rebouissou S, Paris A, Rathahao-Paris E, Perdu E, Bioulac-Sage P, et al. Loss of hepatocyte nuclear factor 1alpha function in human hepatocellular adenomas leads to aberrant activation of signaling pathways involved in tumorigenesis. Hepatology. 2010;51(2):557-66.

6. Buc E, Dupre A, Golffier C, Chabrot P, Flamein $\mathrm{R}$, Dubois A, et al. Positive PET-CT scan in hepatocellular adenoma with concomitant benign liver tumors. Gastroenterol Clin Biol. 2010;34(45):338-41.

7. Slade I, Bacchelli C, Davies H, Murray A, Abbaszadeh F, Hanks S, et al. DICER1 syndrome: clarifying the diagnosis, clinical features and management implications of a pleiotropic tumour predisposition syndrome. Journal of medical genetics. 2011;48(4):273-8. 